\title{
A comparison of resection margins and lymph node clearance in laparoscopic vs open anterior and abdomino-perineal resections for carcinoma of rectum
}

\author{
Galketiya KB ${ }^{1}$, Pinto ${ }^{1}$, Shaminda RAA ${ }^{1}$, Thennakoon TMSUB $^{2}$, Ariyarathna EDKN ${ }^{2}$ \\ ${ }^{\text {I} T e a c h i n g ~ H o s p i t a l, ~ P e r a d e n i y a, ~ S r i ~ L a n k a . ~}$ \\ ${ }^{2}$ Faculty of Medicine, University of Peradeniya, Sri Lanka.
}

Correspondence: Prof.K B Galketiya

e-mail: kbgalketiya@yahoo.com

\begin{abstract}
Introduction: Laparoscopic anterior and abdomino-perineal resections for carcinoma rectum are associated with a lower morbidity than open surgery. It is important to evaluate laparoscopic resections against open surgery for tumour free resection margins and lymph node clearance.
\end{abstract}

Methods: A retrospective analysis of resection margins and number of lymph nodes harvested at anterior and abdomino-perineal resections performed by laparoscopy and open surgery was done.

Results: There was no statistically significant difference in the tumour free resection margins while lymph node clearance was significantly higher in the laparoscopy group.

Conclusion: Laparoscopic surgery for carcinoma rectum provides a satisfactory oncological outcome with regards to clear resection margins and lymph node harvest.

Keywords: Abdomino-perneal resection, laparoscopy, rectal carcinoma

\section{Introduction}

Anterior resection and abdomino-perineal resection are the curative resections for carcinoma of the rectum. Anterior resection is possible when the anus and sphincters can be preserved while having a resection margin of $3 \mathrm{~cm}$. Minimal access resections reduce the length of exposure incision while having a clear vision with magnification facilitating the resection (1). The first laparoscopic assisted left hemicolectomy performed in 1991 reported to have reduced morbidity (2). The problems associated with the new learning curve became less over time, whilst providing clear benefits $(1,3-6)$. The blood loss is lesser as well as post-operative ileus (7). The postoperative morbidity is reduced allowing early mobilization, early feeding and discharge $(1,7)$. Oncologically acceptable resection margins and satisfactory lymph node clearance have also been reported $(7,8)$. This study was designed to evaluate resection margins and lymph node clearance of laparoscopic anterior resections and abdominoperineal resections performed in our unit.

\section{Methods}

A retrospective analysis of resection margins and number of lymph nodes harvested at anterior resection and abdomino-perineal resections performed from January 2014 to April 2016was done and compared with open surgeries performed during the same period. Even though laparoscopic approach is currently preferred in our unit, some patients have to undergo open surgery due to the unavailability of laparoscopy theatre time. 
All laparoscopic resections were performed in standard head low and left lateral tilt. Five ports were used. The dissection was performed with a combination of ultrasonic dissector, bipolar and monopolar diathermy. High ligation of inferior mesenteric artery was performed in all cases. A total mesorectal excision was performed. In case of anterior resection laparoscopic stapled anastomosis was performed. In three anterior resections where tumour was situated at $4 \mathrm{~cm}$ from the anal verge a pull through coloanal anastomosis was done.

Open resections were done in the standard way through the midline laparotomy.

\section{Results}

Thirty four patients were operated during this period and 24 patients underwent laparoscopic procedure. There were twenty anterior resections (LAR) and four abdomino-perineal resections (LAPR) in the laparoscopy group. developed a superficial wound infection but responded to dressings and oral antibiotics.

The results of distal resection margin and lymph node harvest were as follows.

\section{Resection margins}

A comparison of tumour free distal resection margin in laparoscopic anterior resection and open anterior resection is summarised in table 1 . In nineteen out of 20 laparoscopic anterior resections, the distal resection margins were clear. In 17 of laparoscopic resections, the tumour free distal resection margin was more than $3 \mathrm{~cm}$.

In the open surgery group all anterior resections had negative resection margin of $>3 \mathrm{~cm}$. The mean distal resection margins were $4.4 \mathrm{~cm}$ and $4.75 \mathrm{~cm}$ for the laparoscopic and open surgery groups respectively and the difference was not statistically significant $(\mathrm{p}=0.66)$.

Table 1: A comparison of distal resection margin in laparoscopic vs. open anterior resections

\begin{tabular}{lcccc}
\hline Tumour free distal resection margin & $\mathbf{> 5 c m}$ & $\mathbf{3 - 5} \mathbf{c m}$ & $\mathbf{1 - 3} \mathbf{c m}$ & Involved by tumour \\
\hline Laparoscopic & 3 & 14 & 2 & 1 \\
Open & 1 & 5 & 0 & 0 \\
\hline
\end{tabular}

Ten patients underwent open surgery which included six anterior resections (AR) and four abdominoperineal resections (APR).

The mean operating times were as follows; LAR 210min, AR - 220min, LAPR - 200min, APR $180 \mathrm{~min}$. The mean blood loss were; LAR - 100ml, AR - 400ml, LAPR - 150ml, APR - 500ml.

All patients except one were discharged within 10 days. One patient after APR had a prolonged ileus and was discharged on $14^{\text {th }}$ day. One AR patient

\section{Lymph node clearance}

The mean lymph node clearance in laparoscopic and open surgery groups were 20.3 and 17.2 respectively and the difference was statistically significant $(\mathrm{p}<0.001)$.

The number of lymph nodes harvested in laparoscopic vs. open surgery is summarised in table 2).

Table 2: A comparison of lymph node clearance in laparoscopic vs open anterior and abdomino-perineal resections

\begin{tabular}{lccc}
\hline Number of lymph nodes dissected & $\mathbf{1 4 - 1 6}$ & $\mathbf{1 7 - 1 9}$ & $\mathbf{1 9 - 2 1}$ \\
\hline Laparoscopic & 1 & 2 & 21 \\
Open & 3 & 7 & 0 \\
\hline
\end{tabular}




\section{Discussion}

There is an increasing trend for laparoscopic anterior and abdomino-perineal resections worldwide. In most of the studies it is proven that the resection is comparable with open surgery achieving acceptable oncological requirements $(7,8)$ whilst providing benefits of reduced morbidity (1-7). The criteria to assess oncologically acceptable resection, is the number of lymph nodes harvested and tumour free distal resection margin. However, distal resection margin does not apply in abdomino-perineal resections.

In $95 \%(19 / 20)$ of the patients in laparoscopic anterior resection group, the distal resection margin was clear from tumour. In $85 \%$ of them, the distal resection margin was more than $3 \mathrm{~cm}$. The two patients who had tumour free resection margins of $2 \mathrm{~cm}$ and $2.5 \mathrm{~cm}$ underwent a total laparoscopic mobilization, resection and a pull through coloanal anastomosis. The patient who had an involved resection margin was a 72 year old male with a large villous adenoma at $4 \mathrm{~cm}$ from anus. Biopsies showed high grade dysplasia. He underwent laparoscopic resection and pull through coloanal anastomosis as per anal resection was attempted and failed. His histopathology revealed an adenocarcinoma within a villous adenoma and the resection margin showed high grade dysplasia. He was treated with post operative chemo-irradiation and being followed up.

In contrast, all six patients who underwent open anterior resections had a tumour free distal resection margin of more than $3 \mathrm{~cm}$. However in all of them tumours were located more than $5 \mathrm{~cm}$ from anal verge. According to our findings there was no statistically significant difference in tumour free distal resection margin between the laparoscopic and open surgery groups.

The mean lymph node clearance in laparoscopic surgery was significantly higher than that of open surgery performed in our unit. Pechlivanides G, et al has shown in their study that laparoscopic resection of the rectum can achieve similar lymph node clearance to the open approach (8).

By performing a high ligation of inferior mesenteric vessels and a total mesorectal excision it is possible to achieve the oncological principles of resection. A clear advantage of laparoscopic resection is the clarity of vision provided by magnification and zooming closer to area of dissection (1). The combination of ultrasonic dissector, bipolar and monopolar diathermy allows a dissection with minimal blood loss.

\section{Conclusions}

Laparoscopic anterior resection and abdominoperineal resection have provided satisfactory tumour clearance with negative resection margins in a large majority of cases. Even though open surgery achieved clear resection margins in all patients operated, the difference was not statistically significant. Meanwhile, the lymph node clearance was significantly higher in laparoscopic surgery group comparable to open surgery group.

\section{References}

1. Beat M Kunzil, Helmut Friess, Shailesh V Shrikhande. Is laparoscopic colorectal cancer surgery equal to open surgery? An evidence based perspective. World Journal of Gastrointestinal Surgery 2010; 2(4): 101-8.

2. Redwine DB, Sharpe DR. Laparoscopic segmental resection of the sigmoid colon for endometriosis. JLaparoendoscopic Surg 1991; 1(4): 217-20.

3. Monson JR, Hill AD, Darzi A. Laparoscopic colonic surgery. British Journal of Surgery 1995; 82(2): 150-7.

4. Decanini C, Milson JW, Bohm B, Fazio VW. Laparoscopic oncologic abdomino-perineal resection. Dis Colon Rectum 1994; 37(6): 552-8.

5. Ziprin P, Ridgway PF, Peck DH, Darzi AW. The theories and realities of port site metastases: a critical appraisal. JAm Coll Surg 2002; 195(3): 395-498.

6. Stocchi L, Nelson H, Young-fadok TM, et al. Safety and advantages of laparoscopic vs open colectomy in the elderly, matched control study. Dis Colon Rectum 2000; 43(3): 326-32.

7. MM Reza, JA Blasco, E Andradas, R Cantero, J Mayol. Systematic review of laparoscopic versus open surgery for colorectal cancer. British Journal of Surgery 2006; 93: 921-28.

8. George Pechlivanides, Nicolaos Gouvas, John Tsiaoussis, et al. Lymph node clearance after total mesorectal excision for rectal cancer: Laparoscopic versus open approach. Digestive diseases 2007; 25: 94-99. 